\title{
SPECTROSCOPY OF $\Omega_{c c}, \Omega_{b b}$ AND $\Omega_{b c}$ BARYONS IN HYPERCENTRAL CONSTITUENT QUARK MODEL VIA ANSATZ METHOD
}

\author{
NASRIN SALEHI \\ Department of Basic Sciences, Shahrood Branch, Islamic Azad University \\ Shahrood, Iran \\ salehi@shahroodut.ac.ir
}

(Received August 8, 2018; accepted February 27, 2019)

In this paper, we exhibited the ground and excited state masses of doubly heavy $\Omega$ baryons. For this purpose, we have analytically solved the sixdimensional radial Schrödinger equation for three identical particles with the hypercentral potential by using the Ansatz method. The hypercentral potential is considered as a combination of the hypercoulomb, linear confining, and the harmonic oscillator terms which has a two-body character and turns out to be exactly hypercentral. We also incorporated the first order correction and the spin-dependent part to the confinement potential. Our calculations have been performed for the radial excited states as well as orbital excited states masses of $\Omega_{c c}, \Omega_{b b}$ and $\Omega_{b c}$ baryons. The obtained masses are compared with other theoretical predictions, which could be a useful tool for the interpretation of experimentally unknown doubly heavy baryons spectrum.

DOI:10.5506/APhysPolB.50.735

\section{Introduction}

Baryons are strongly interacting fermions made up of three quarks. Singly heavy baryons have one heavy quark and two light quarks. Doubly heavy baryons, with two heavy quarks and one light quark, are expected to exist in QCD and their masses have been predicted in the quark model. $\Omega$ baryons have a light strange quark with two heavy quarks $(c$ and $b)$ [1]. In the last years, experimental and theoretical outcomes have been used in studying the heavy baryons. A lot of new experimental results have been reported by different collaborations including CLEO, Belle, BaBar and LHCb [2, 3] on ground and many new excited states of heavy flavour baryons. The Particle Data Group (PDG) listed 20 known charm baryons [4]. The experimental evidence comes for $\Xi_{c c}^{+}$(containing two charm quarks) with a mass 
of $\sim 3520 \mathrm{MeV} / c^{2}$ by the SELEX experiment, and LHCb has determined the five excited states of $\Omega_{c}$ baryon and the ground state of $\Xi_{c c}^{+}$baryon [4-7]. Recently, various phenomenological approaches have been used to study the doubly heavy baryons including relativistic quark model [8], the chiral unitary model [9], the extended local hidden gauge approach [10], the relativistic fluxtube (RFT) model [11], the Hamiltonian model [12], Regge phenomenology [13], QCD sum rule [14, 15], etc.

The nonrelativistic constituent quark models (CQM) among them have also yielded good results studying the baryons static properties [16, 17], such as the baryon spectrum $[1,8,12,18-20]$, the magnetic moments, the photocouplings [21, 22], the electromagnetic form factors [23] and the strong decay amplitudes. The spectrum of baryon is usually well-described. However, the various models are quite different. It is necessary to note that the study of hadron spectroscopy is not enough to distinguish the different forms of quark dynamics. To do so, we need to study in a consistent way all the physical observables of interest. This systematic study of baryon properties is better done using a general framework. Here, a hypercentral approach to quark dynamics can be applied [24, 25]. As proposed by lattice QCD calculations [26, 27], the model comprises a hypercentral quark interaction involving a linear plus Coulomb-like term. The hCQM scheme can be used for baryons, which is an average two-body potential for the three-quark system over the hyperangle and performs quite well. Our study here is also based on the hypercentral constituent quark model (hCQM). Since the solution of the hyper-radial Schrödinger equation with the Cornell potential cannot be obtained analytically [23], therefore, we added the harmonic oscillator terms which has a two-body character and turns out to be exactly hypercentral $[2,4,18]$. In fact, we have used a modified version of the original model with only hypercoulomb and linear confinement in the hyper-radious [2]. We also added the first order correction and the spin-dependent part to the potential, and calculations for doubly heavy $\Omega$ baryons masses have been performed by solving six-dimensional hyper-radial Schrödinger equations by using the Ansatz method. We have obtained the mass spectra of radial excited states up to $5 S$ and orbital excited states for $1 P-5 P, 1 D-4 D$ and $1 F-2 F$ states.

This paper is organized as follows: after the introduction of doubly heavy baryons, the hCQM and the interaction potentials between three quarks in baryons are explained in Sec. 2. In Sec. 3, we present the quasi-exact analytical solution of the radial Schrödinger equation for our proposed potential. In Sec. 4, our masses spectra results are given compared with other predictions. Our concluding remarks are given in Sec. 5 . 


\section{The hypercentral model and hypercentral potential}

In this paper, the hypercentral Constitute Quark Model (hCQM) has been used to generate the mass spectrum of doubly heavy baryons. The brief description of hCQM model is as follows.

By considering baryon as a three-body system, in the center-of-mass frame $\left(R_{\mathrm{cm}}=0\right)$, the internal quark motion is described by the Jacobi coordinates, $\rho$ and $\lambda[28]$, defined as:

$$
\vec{\rho}=\frac{1}{\sqrt{2}}\left(\vec{r}_{1}-\vec{r}_{2}\right), \quad \vec{\lambda}=\frac{m_{1} \vec{r}_{1}+m_{2} \vec{r}_{2}-\left(m_{1}+m_{2}\right) \vec{r}_{3}}{\sqrt{m_{1}^{2}+m_{2}^{2}+\left(m_{1}+m_{2}\right)^{2}}} .
$$

The respective reduced masses are given by

$$
m_{\rho}=\frac{2 m_{1} m_{2}}{m_{1}+m_{2}}, \quad m_{\lambda}=\frac{2 m_{3}\left(m_{1}^{2}+m_{2}^{2}+m_{1} m_{2}\right)}{\left(m_{1}+m_{2}\right)\left(m_{1}+m_{2}+m_{3}\right)} .
$$

Here, $m_{1}, m_{2}$ and $m_{3}$ are the constituent quark masses.

The angles of the hyperspherical coordinates are given by $\Omega_{\rho}=\left(\theta_{\rho}, \phi_{\rho}\right)$ and $\Omega_{\lambda}=\left(\theta_{\lambda}, \phi_{\lambda}\right)$. In order to describe the three-quark dynamics, we define hyper-radius $x$ and hyperangle $\xi$ in terms of the absolute values $\rho$ and $\lambda$ of the Jacobi coordinates [29] as

$$
x=\sqrt{\rho^{2}+\lambda^{2}}, \quad \xi=\arctan \left(\frac{\rho}{\lambda}\right) .
$$

In the present paper, the confining three-body potential is regarded as a combination of three hypercentral interacting potentials. First, the sixdimensional hypercoulomb potential $[30,31]$ which is attractive for small separations

$$
V_{\text {hyc }}(x)=\frac{\tau}{x},
$$

while at large separations, a hyperlinear term gives rise to quark confinement $[1,2]$

$$
V_{\text {com }}=\beta x .
$$

$\beta$ corresponds to the string tension of the confinement [32]. Third, the sixdimensional harmonic oscillator potential, which has a two-body character, and turns out to be exactly hypercentral since [33]

$$
V_{\mathrm{ho}}=\sum_{i<j}^{i=3} \frac{1}{2} k\left(r_{i}-r_{j}\right)^{2}=\frac{3}{2} k x^{2}=p x^{2} .
$$


The first order correction $V^{(1)}(x)$ can be written as [33-35]

$$
V^{(1)}(x)=-C_{\mathrm{F}} C_{\mathrm{A}} \frac{\alpha_{\mathrm{s}}^{2}}{4 x^{2}} .
$$

The parameters $C_{\mathrm{F}}=\frac{2}{3}$ and $C_{\mathrm{A}}=3$ are the Casimir charges of the fundamental and adjoint representation. The hypercoulomb strength $\tau=-\frac{2}{3} \alpha_{\mathrm{s}}$, $\frac{2}{3}$ is the color factor for the baryon. $\alpha_{\mathrm{s}}$ is the strong running coupling constant, which is written as

$$
\alpha_{\mathrm{S}}=\frac{\alpha_{\mathrm{s}}\left(\mu_{0}\right)}{1+\left(\frac{33-2 n_{\mathrm{f}}}{12 \pi}\right) \alpha_{\mathrm{S}}\left(\mu_{0}\right) \ln \left(\frac{m_{1}+m_{2}+m_{3}}{\mu_{0}}\right)} .
$$

The spin-dependent part $V_{\mathrm{SD}}(x)$ is given as

$V_{\mathrm{SD}}(x)=V_{\mathrm{SS}}(x)\left(\vec{S}_{\rho} \cdot \vec{S}_{\lambda}\right)+V_{\gamma \mathrm{S}}(x)(\vec{\gamma} \cdot \vec{S})+V_{\mathrm{T}}(x)\left[S^{2}-\frac{3(\vec{S} \cdot \vec{x})(\vec{S} \cdot \vec{x})}{x^{2}}\right]$.

The spin-dependent potential, $V_{\mathrm{SD}}(x)$ contains three types of the interaction terms [36] including the spin-spin term $V_{\mathrm{SS}}(x)$, the spin-orbit term $V_{\gamma \mathrm{S}}(x)$ and tensor term $V_{\mathrm{T}}(x)$ described in Ref. [37]. Here, $S=S_{\rho}+S_{\lambda}$, where $S_{\rho}$ and $S_{\lambda}$ are the spin vectors associated with the $\rho$ and $\lambda$ variables, respectively. The coefficient of these spin-dependent terms can be written in terms of the vector, $V_{\mathrm{V}}(x)=\frac{\tau}{x}$ and scalar, $V_{\mathrm{S}}(x)=\beta x+p x^{2}$ parts of the static potential as [29]

$$
\begin{aligned}
V_{\gamma \mathrm{S}} & =\frac{1}{2 m_{\rho} m_{\lambda} x}\left(3 \frac{\mathrm{d} V_{\mathrm{V}}}{\mathrm{d} x}-\frac{\mathrm{d} V_{\mathrm{S}}}{\mathrm{d} x}\right), \\
V_{\mathrm{T}}(x) & =\frac{1}{6 m_{\rho} m_{\lambda}}\left(\frac{3 \mathrm{~d}^{2} V_{\mathrm{V}}}{\mathrm{d}^{2} x}-\frac{1}{x} \frac{\mathrm{d} V_{\mathrm{V}}}{\mathrm{d} x}\right), \\
V_{\mathrm{SS}}(x) & =\frac{1}{3 m_{\rho} m_{\lambda}} \nabla^{2} V_{\mathrm{V}} .
\end{aligned}
$$

In our model, the hypercentral interaction potential is assumed as follows [38]:

$$
V(x)=V^{(0)}(x)+\left(\frac{1}{m_{\rho}}+\frac{1}{m_{\lambda}}\right) V^{(1)}(x)+V_{\mathrm{SD}}(x),
$$

where $V^{(0)}(x)$ is given by

$$
\begin{aligned}
& V^{(0)}(x)=V_{\mathrm{hyc}}(x)+V_{\mathrm{con}}(x)+V_{\mathrm{ho}}(x), \\
& V^{(0)}(x)=\frac{\tau}{x}+\beta x+p x^{2} .
\end{aligned}
$$


In our purposed hypercentral potential, we have six parameters: $m_{1}, m_{2}$, $m_{3}, \tau, \beta$ and $p$. The baryon masses are determined by the sum of the model quark masses plus kinetic energy, potential energy and the spin-dependent interaction as [39]

$$
M_{B}=\sum_{i=1}^{3} m_{i}+\langle H\rangle .
$$

First, we will solve the Schrödinger equation via the quasi-exact analytical Ansatz approach and obtain the corresponding eigenvalues.

\section{Quasi-exact analytical solution of the six-dimensional radial Schrödinger equation via Ansatz approach}

The Hamiltonian of the three-body baryonic system in the hypercentral constituent quark model is expressed as [40]

$$
H=\frac{P_{\rho}^{2}}{2 m}+\frac{P_{\lambda}^{2}}{2 m}+V(x)
$$

and the hyper-radial wave function $\psi_{\nu \gamma}(x)$ is determined by the hypercentral Schrödinger equation. The hyper-radial Schrödinger equation corresponding to the above Hamiltonian can be written as [1, 2, 38]

$$
\left(\frac{\mathrm{d}^{2}}{\mathrm{~d} x^{2}}+\frac{5}{x} \frac{\mathrm{d}}{\mathrm{d} x}-\frac{\gamma(\gamma+4)}{x^{2}}\right) \psi_{\nu \gamma}(x)=-2 m[E-V(x)] \psi_{\nu \gamma}(x),
$$

where $\gamma$ is the grand angular quantum number given by $\gamma=2 n+l_{\rho}+l_{\lambda}$, $n=0,1, \ldots ; l_{\rho}$ and $l_{\lambda}$ are the angular momenta associated with the $\vec{\rho}$ and $\vec{\lambda}$ variables. $\nu$ denotes the number of nodes of the space three-quark wave function [41]. In Eq. (18), $m$ is the reduced mass [42] which is defined as

$$
m=\frac{2 m_{\rho} m_{\lambda}}{m_{\rho}+m_{\lambda}} .
$$

The transformation [1, 2]

$$
\psi_{\nu \gamma}(x)=x^{-\frac{5}{2}} \phi_{\nu \gamma}
$$

reduces Eq. (18) to the form of

$$
\begin{aligned}
& \phi_{\nu \gamma}^{\prime \prime}(x)+\left[\varepsilon-r_{1} x^{2}-r_{2} x-\frac{r_{3}}{x}-\frac{r_{4}}{x^{2}}-\frac{r_{5}}{x^{3}}+\frac{r_{6}}{x^{5}}+r_{7}-\frac{(2 \gamma+3)(2 \gamma+5)}{4 x^{2}}\right] \\
& \times \phi_{\nu \gamma}(x)=0 .
\end{aligned}
$$


The hyper-radial wave function $\phi_{\nu \gamma}(x)$ is a solution of the reduced Schrödinger equation for each of the three identical particles with the mass $m$ and interacting potential (13), where

$$
\begin{aligned}
\varepsilon & =2 m E, \quad r_{1}=2 m p, \quad r_{2}=2 m \beta, \quad r_{3}=2 m \tau \\
r_{4} & =2 m\left(\frac{1}{m_{\rho}}+\frac{1}{m_{\lambda}}\right)\left(-C_{\mathrm{F}} C_{\mathrm{A}} \frac{\alpha_{\mathrm{s}}^{2}}{4}\right), \\
r_{5} & =2 m\left[\frac{2 \tau}{3 m_{\rho} m_{\lambda}}\left(S_{\rho} \cdot S_{\lambda}\right)-\frac{3 \tau}{2 m_{\rho} m_{\lambda}}(\vec{\gamma} \cdot \vec{s})+\frac{7 \tau}{6 m_{\rho} m_{\lambda}} s^{2}\right], \\
r_{6} & =2 m \frac{21 \tau}{6 m_{\rho} m_{\lambda}}(\vec{s} \cdot \vec{x})(\vec{s} \cdot \vec{x}), \\
r_{7} & =2 m\left(\frac{(\beta+2 p)}{2 m_{\rho} m_{\lambda}}(\vec{\gamma} \cdot \vec{s})\right) .
\end{aligned}
$$

We assume the following form for the wave function:

$$
\phi_{\nu \gamma}=h(x) e^{g(x)} .
$$

Now, we make use of the Ansatz for the $h(x)$ and $g(x)$ [43-45]

$$
\begin{array}{rlrl}
h(x) & =\Pi\left(x-\alpha_{i}^{\nu}\right), & \nu=1,2, \ldots, \\
h(x) & =1, & \nu=0, \\
g(x) & =a \ln x+q x^{2}+c x+\frac{d}{x},
\end{array}
$$

where $a, q, c$ and $d$ are positive constants. From Eq. (23), we obtain

$$
\phi^{\prime \prime}(x)=\left[g^{\prime \prime}(x)+g^{\prime 2}(x)+\left(\frac{h^{\prime \prime}(x)+2 h^{\prime}(x) g^{\prime}(x)}{h(x)}\right)\right] \phi(x) .
$$

Comparing Eqs. (21) and (25), it can be found that

$$
\begin{aligned}
& {\left[r_{1} x^{2}+r_{2} x+\frac{r_{3}}{x}+\frac{r_{4}}{x^{2}}+\frac{r_{5}}{x^{3}}-\frac{r_{6}}{x^{5}}-r_{7}+\frac{(2 \gamma+3)(2 \gamma+5)}{4 x^{2}}-\varepsilon\right]} \\
& =\left[g^{\prime \prime}(x)+g^{\prime 2}(x)+\frac{h^{\prime \prime}(x)+2 h^{\prime}(x) g^{\prime}(x)}{h(x)}\right] .
\end{aligned}
$$

By substituting Eq. (24) into Eq. (26), we obtain the following equation:

$$
\begin{aligned}
& -\varepsilon+r_{1} x^{2}+r_{2} x+\frac{r_{3}}{x}+\frac{r_{4}}{x^{2}}+\frac{r_{5}}{x^{3}}-\frac{r_{6}}{x^{5}}-r_{7}+\frac{(2 \gamma+3)(2 \gamma+5)}{4 x^{2}} \\
& =4 q^{2} x^{2}+4 c q x+\frac{(2 a c-4 d q)}{x}+\frac{\left(a^{2}-a-2 c d\right)}{x^{2}}+\frac{2 d(1-a)}{x^{3}}+\frac{d^{2}}{x^{4}} \\
& \quad+\left(c^{2}+2 q+4 a c\right) .
\end{aligned}
$$


By equating the corresponding powers of $x$ on both sides of Eq. (27), we can obtain

$$
\begin{aligned}
& a=\frac{2 \tau}{\beta} \sqrt{\frac{m p}{2}}, \quad c=\frac{m \beta}{2} \sqrt{\frac{2}{m p}}, \quad q=\sqrt{\frac{m p}{2}} \\
& \varepsilon=-\left[\frac{m \beta^{2}}{2 p}+2 \sqrt{\frac{m p}{2}}+\frac{4 m p \tau}{\beta}+2 m\left(\frac{(\beta+2 p)}{2 m_{\rho} m_{\lambda}}(\vec{\gamma} \cdot \vec{s})\right)\right] .
\end{aligned}
$$

Since $p=\frac{m \omega^{2}}{2}$, we have

$$
a=\frac{2 m \omega}{2 \beta}, \quad c=\frac{\beta}{\omega}, \quad q=\frac{m \omega}{2} .
$$

The energy eigenvalues for the mode $\nu=0$ and grand angular momentum $\gamma$ from Eqs. (22) and (28) are given as follows:

$$
E=-\left[\frac{\beta^{2}}{2 m \omega}+\frac{\omega}{2}+\frac{m \omega^{2} \tau}{\beta}+\left(\frac{\left(\beta+m \omega^{2}\right)}{2 m_{\rho} m_{\lambda}}\right)(\vec{\gamma} \cdot \vec{s})\right]
$$

Finally, for calculating the best doubly heavy baryons masses $\left(\Omega_{c c}, \Omega_{b b}\right.$, $\Omega_{b c}$ ) predictions, the values of $m_{s}, m_{c}, m_{b}, \alpha_{\mathrm{s}}, \omega$ and $\beta$ (which are listed in Table I) are selected using genetic algorithm. The cost function of genetic algorithms is the minimum difference between our calculated baryon mass and the reported baryons mass of other works.

TABLE I

The quark mass (in $\mathrm{GeV}$ ) and the fitted values of the parameters used in our calculations.

\begin{tabular}{cccccccc}
\hline \hline$m_{s}$ & $m_{c}$ & $m_{b}$ & $\alpha_{\mathrm{s}}$ & $C_{\mathrm{F}}$ & $C_{\mathrm{A}}$ & $\beta$ & $\omega$ \\
\hline 0.565 & 1.345 & 4.902 & 0.340 & $\frac{2}{3}$ & 3 & 0.01 & $0.142 \mathrm{fm}^{-1}$
\end{tabular}

\section{Results and discussions: mass spectrum}

The ground and excited states of doubly heavy $\Omega$ baryons are unknown to us experimentally. Hence, we have obtained the ground and excited state masses of $\Omega_{c c}, \Omega_{b b}$ and $\Omega_{b c}$ (Tables II, III, IV, V and VI). These mass spectra are estimated by using the hypercentral potential Eq. (13) in the hypercentral constituent quark model. We begin with the ground state $1 S$ and the masses are computed for both parities $J^{P}=\frac{1}{2}^{+}$and $J^{P}=\frac{3}{2}^{+}$. Our predicted ground state masses of doubly heavy $\Omega$ baryons are compared with 
other predictions in Table II. Our calculations for the ground state masses of $\Omega_{c c}^{+}, \Omega_{b b}^{-}$and $\Omega_{b c}^{0}$ are different from other predictions in the vicinity of $\approx 100 \mathrm{MeV}, \approx 500 \mathrm{MeV}$ and $\approx 400 \mathrm{MeV}$, respectively.

TABLE II

The ground state masses of $\Omega_{c c}^{+}, \Omega_{b b}^{-}$and $\Omega_{b c}^{0}$ are listed with other theoretical predictions (in $\mathrm{GeV}$ ).

\begin{tabular}{l|cc|cc|cc}
\hline \hline \multicolumn{1}{c|}{ Baryons } & \multicolumn{2}{|c|}{$\Omega_{c c}^{+}$} & \multicolumn{2}{|c|}{$\Omega_{b b}^{-}$} & \multicolumn{2}{c}{$\Omega_{b c}^{0}$} \\
\hline \multicolumn{1}{c|}{$J^{P}$} & $\frac{1}{2}^{+}$ & $\frac{3}{2}^{+}$ & $\frac{1}{2}^{+}$ & $\frac{3}{2}^{+}$ & $\frac{1}{2}^{+}$ & $\frac{3}{2}^{+}$ \\
\hline Our calc. & 3.662 & 3.677 & 10.870 & 10.866 & 7.329 & 7.339 \\
Ref. [38] & 3.650 & 3.810 & 10.446 & 10.467 & 7.136 & 7.187 \\
Ref. [46] & 3.719 & 3.746 & 10.442 & 10.432 & 6.999 & 7.024 \\
Ref. [47] & 3.778 & 3.872 & 10.359 & 10.389 & 7.088 & 7.130 \\
Ref. [48] & 3.648 & 3.770 & 10.271 & 10.289 & 6.994 & 7.017 \\
Refs. [49, 50] & 3.710 & 3.760 & 10.320 & 10.380 & & \\
Refs. [51, 52] & 3.730 & 3.780 & 9.970 & 10.50 & 6.750 & 7.300 \\
Ref. [53] & 3.832 & 3.883 & 10.447 & 10.467 & & \\
Ref. [54] & 3.815 & 3.876 & 10.454 & 10.486 & 7.136 & 7.187 \\
Ref. [55] & 3.697 & 3.769 & 10.293 & 10.321 & & \\
Ref. [56] & 3.747 & 3.819 & & & & \\
Ref. [57] & 3.713 & 3.785 & & & & \\
Ref. [58] & 3.738 & 3.822 & 10.273 & 10.308 & 6.999 & 7.059 \\
Ref. [59] & 3.740 & 3.779 & & & & \\
Ref. [60] & 3.654 & 3.724 & & & & \\
Refs. [61, 62] & 3.650 & 3.809 & 10.320 & 10.430 & & \\
Ref. [63] & 3.702 & 3.783 & 10.260 & 10.297 & 6.986 & 7.046 \\
Ref. [64] & 3.667 & 3.758 & 10.397 & 10.495 & 7.103 & 7.200 \\
Ref. [65] & 3.710 & 3.800 & 10.208 & 10.244 & 6.999 & 7.063 \\
Ref. [66] & 3.740 & 3.820 & 10.370 & 10.400 & 7.045 & 7.120
\end{tabular}

The radial excited state masses for these three doubly heavy baryons are computed from $2 S-5 S$ and are compared with Refs. [38, 47, 48, 53-55] in Table III.

We can observe that our $2 S$ and $3 S$ states show a smaller difference in $\mathrm{MeV}$, see Refs. [38, 47], than other references, and for $4 S$ and $5 S$ states, have a difference in the range of $\approx 100 \mathrm{MeV}$ comparing with Ref. [38] for $\Omega_{c c}^{+}$. Our calculations for $2 S$ and $3 S$ states of $\Omega_{b b}^{-}$are close to Refs. [38, 48] and for $4 S$ and $5 S$ states, have a difference in the range of $\approx 150 \mathrm{MeV}$ comparing with Ref. [38]. In the case of $\Omega_{b c}$, we can observe that our $2 S, 3 S, 4 S$ and $5 S$ state masses with $J^{P}=\frac{1}{2}^{+}$are $5 \mathrm{MeV}, 158 \mathrm{MeV}, 243 \mathrm{MeV}$ and $267 \mathrm{MeV}$, while $12 \mathrm{MeV}, 156 \mathrm{MeV}, 236 \mathrm{MeV}$ and $261 \mathrm{MeV}$ (with $J^{P}=\frac{3}{2}^{+}$) are lower than the results of Ref. [38], respectively. 
The masses of radial excited states for $\Omega_{c c}, \Omega_{b b}$ and $\Omega_{b c}$ (in GeV).

\begin{tabular}{|c|c|c|c|c|c|c|c|c|c|}
\hline Baryons & State & $J^{P}$ & Our calc. & [38] & [53] & [54] & [55] & [48] & {$[47]$} \\
\hline \multirow[t]{8}{*}{$\Omega_{c c}$} & \multirow[t]{2}{*}{$2 S$} & \multirow{2}{*}{$\begin{array}{l}\frac{1}{2}^{+} \\
\frac{3}{2}^{+}\end{array}$} & 3.964 & 4.041 & 4.227 & 4.180 & 4.112 & 4.268 & 4.075 \\
\hline & & & 3.979 & 4.096 & 4.263 & 4.188 & 4.160 & 4.334 & 4.174 \\
\hline & \multirow[t]{2}{*}{$3 S$} & $\frac{1}{2}^{+}$ & 4.177 & 4.338 & 4.295 & & & 4.714 & 4.321 \\
\hline & & $\frac{3}{2}^{+}$ & 4.193 & 4.365 & 4.265 & & & 4.776 & \\
\hline & \multirow[t]{2}{*}{$4 S$} & $\frac{1}{2}^{+}$ & 4.452 & 4.598 & & & & & \\
\hline & & $\frac{3}{2}^{+}$ & 4.467 & 4.614 & & & & & \\
\hline & \multirow[t]{2}{*}{$5 S$} & $\frac{1}{2}^{+}$ & 4.787 & 4.836 & & & & & \\
\hline & & $\frac{3}{2}^{+}$ & 4.802 & 4.845 & & & & & \\
\hline \multirow[t]{8}{*}{$\Omega_{b b}$} & \multirow[t]{2}{*}{$2 S$} & $\frac{1}{2}^{+}$ & 10.969 & 10.736 & 10.707 & 10.693 & 10.604 & 10.830 & 10.610 \\
\hline & & $\frac{3}{2}^{+}$ & 10.964 & 10.743 & 10.723 & 10.721 & 10.622 & 10.839 & 10.645 \\
\hline & \multirow[t]{2}{*}{$3 S$} & $\frac{1}{2}^{+}$ & 11.036 & 10.983 & 10.744 & & & 11.240 & 10.806 \\
\hline & & $\frac{3}{2}^{+}$ & 11.032 & 10.986 & 10.730 & & & 11.247 & 10.843 \\
\hline & \multirow[t]{2}{*}{$4 S$} & $\frac{1}{2}^{+}$ & 11.123 & 11.205 & 10.994 & & & & \\
\hline & & $\frac{3}{2}^{+}$ & 11.119 & 11.207 & 11.031 & & & & \\
\hline & \multirow[t]{2}{*}{$5 S$} & $\frac{1}{2}^{+}$ & 11.230 & 11.411 & & & & & \\
\hline & & $\frac{3}{2}^{+}$ & 11.225 & 11.412 & & & & & \\
\hline \multirow[t]{8}{*}{$\Omega_{b c}$} & \multirow[t]{2}{*}{$2 S$} & $\frac{1}{2}^{+}$ & 7.475 & 7.480 & & & & 7.559 & \\
\hline & & $\frac{3}{2}^{+}$ & 7.485 & 7.497 & & & & 7.571 & \\
\hline & \multirow[t]{2}{*}{$3 S$} & $\frac{1}{2}^{+}$ & 7.609 & 7.767 & & & & 7.976 & \\
\hline & & $\frac{3}{2}^{+}$ & 7.619 & 7.775 & & & & 7.985 & \\
\hline & \multirow[t]{2}{*}{$4 S$} & $\frac{1}{2}^{+}$ & 7.782 & 8.023 & & & & & \\
\hline & & $\frac{3}{2}^{+}$ & 7.792 & 8.028 & & & & & \\
\hline & \multirow[t]{2}{*}{$5 S$} & $\frac{1}{2}^{+}$ & 7.993 & 8.260 & & & & & \\
\hline & & $\frac{3}{2}^{+}$ & 8.002 & 8.263 & & & & & \\
\hline
\end{tabular}

To calculate the orbital excited state masses $(1 P-5 P, 1 D-4 D, 1 F-2 F)$, we have considered all possible isospin splitting and all combinations of total spin $S$ and total angular momentum $J$. Our outcomes and the comparison of masses with other approaches are also tabulated in Tables IV, V and VI. 
TABLE IV

The masses of orbital excited states of $\Omega_{c c}$ (in $\mathrm{GeV}$ ).

\begin{tabular}{|c|c|c|c|c|c|c|c|c|}
\hline State & Our calc. & [38] & [53] & [54] & [57] & {$[47]$} & [61] & Other \\
\hline$\left(1^{2} P_{1 / 2}\right)$ & 3.965 & 3.989 & 4.086 & 4.046 & 4.061 & 4.002 & & $4.009[55]$ \\
\hline$\left(1^{2} P_{3 / 2}\right)$ & 3.956 & 3.972 & 4.086 & 4.052 & 4.132 & 4.102 & 3.910 & \\
\hline$\left(1^{4} P_{1 / 2}\right)$ & 3.987 & 3.998 & & & & & & \\
\hline$\left(1^{4} P_{3 / 2}\right)$ & 3.978 & 3.981 & & & & & & $3.960[52]$ \\
\hline$\left(1^{4} P_{5 / 2}\right)$ & 3.926 & 3.958 & 4.220 & 4.152 & & & 4.058 & \\
\hline$\left(2^{2} P_{1 / 2}\right)$ & 4.194 & 4.273 & 4.199 & 4.135 & & 4.251 & & $4.101[55]$ \\
\hline$\left(2^{2} P_{3 / 2}\right)$ & 4.155 & 4.259 & 4.201 & 4.140 & & 4.345 & & \\
\hline$\left(2^{4} P_{1 / 2}\right)$ & 4.215 & 4.280 & & & & & & \\
\hline$\left(2^{4} P_{3 / 2}\right)$ & 4.176 & 4.266 & & & & & & \\
\hline$\left(2^{4} P_{5 / 2}\right)$ & 4.154 & 4.247 & & & & & & \\
\hline$\left(3^{2} P_{1 / 2}\right)$ & 4.453 & 4.529 & & & & & & \\
\hline$\left(3^{2} P_{3 / 2}\right)$ & 4.444 & 4.517 & & & & & & \\
\hline$\left(3^{4} P_{1 / 2}\right)$ & 4.475 & 4.536 & & & & & & \\
\hline$\left(3^{4} P_{3 / 2}\right)$ & 4.466 & 4.523 & & & & & & \\
\hline$\left(3^{4} P_{5 / 2}\right)$ & 4.414 & 4.506 & & & & & & \\
\hline$\left(4^{2} P_{1 / 2}\right)$ & 4.803 & 4.767 & & & & & & \\
\hline$\left(4^{2} P_{3 / 2}\right)$ & 4.764 & 4.755 & & & & & & \\
\hline$\left(4^{4} P_{1 / 2}\right)$ & 4.825 & 4.772 & & & & & & \\
\hline$\left(4^{4} P_{3 / 2}\right)$ & 4.788 & 4.761 & & & & & & \\
\hline$\left(4^{4} P_{5 / 2}\right)$ & 4.763 & 4.745 & & & & & & \\
\hline$\left(5^{2} P_{1 / 2}\right)$ & 5.183 & 4.989 & & & & & & \\
\hline$\left(5^{2} P_{3 / 2}\right)$ & 5.175 & 4.978 & & & & & & \\
\hline$\left(5^{4} P_{1 / 2}\right)$ & 5.206 & 4.994 & & & & & & \\
\hline$\left(5^{4} P_{3 / 2}\right)$ & 5.198 & 4.984 & & & & & & \\
\hline$\left(5^{4} P_{5 / 2}\right)$ & 5.145 & 4.969 & & & & & & \\
\hline$\left(1^{4} D_{1 / 2}\right)$ & 4.215 & 4.186 & & & & & & \\
\hline$\left(1^{2} D_{3 / 2}\right)$ & 4.156 & 4.162 & & & & & & \\
\hline$\left(1^{4} D_{3 / 2}\right)$ & 4.193 & 4.170 & & & & & & \\
\hline$\left(1^{2} D_{5 / 2}\right)$ & 4.116 & 4.141 & 4.264 & 4.202 & & & 4.153 & \\
\hline$\left(1^{4} D_{5 / 2}\right)$ & 4.155 & 4.149 & & & & & & \\
\hline$\left(1^{4} D_{7 / 2}\right)$ & 4.086 & 4.122 & & & & & 4.294 & \\
\hline$\left(2^{4} D_{1 / 2}\right)$ & 4.490 & 4.446 & & & & & & \\
\hline$\left(2^{2} D_{3 / 2}\right)$ & 4.429 & 4.425 & & & & & & \\
\hline$\left(2^{4} D_{3 / 2}\right)$ & 4.468 & 4.432 & & & & & & \\
\hline$\left(2^{2} D_{5 / 2}\right)$ & 4.391 & 4.407 & & & & & & \\
\hline$\left(2^{4} D_{5 / 2}\right)$ & 4.430 & 4.414 & 4.299 & 4.232 & & & & \\
\hline$\left(2^{4} D_{7 / 2}\right)$ & 4.360 & 4.391 & & & & & & \\
\hline
\end{tabular}


Continued.

\begin{tabular}{ccccccccc}
\hline \hline State & Our calc. & {$[38]$} & {$[53]$} & {$[54]$} & {$[57]$} & {$[47]$} & {$[61]$} & Other \\
\hline$\left(3^{4} D_{1 / 2}\right)$ & 4.823 & 4.642 & & & & & & \\
$\left(3^{2} D_{3 / 2}\right)$ & 4.764 & 4.625 & & & & & & \\
$\left(3^{4} D_{3 / 2}\right)$ & 4.802 & 4.631 & & & & & & \\
$\left(3^{2} D_{5 / 2}\right)$ & 4.726 & 4.611 & & & & & & \\
$\left(3^{4} D_{5 / 2}\right)$ & 4.763 & 4.616 & 4.410 & & & & & \\
$\left(3^{4} D_{7 / 2}\right)$ & 4.695 & 4.598 & & & & & & \\
$\left(4^{4} D_{1 / 2}\right)$ & 5.221 & 4.911 & & & & & & \\
$\left(4^{2} D_{3 / 2}\right)$ & 5.160 & 4.894 & & & & & & \\
$\left(4^{4} D_{3 / 2}\right)$ & 5.198 & 4.900 & & & & & \\
$\left(4^{2} D_{5 / 2}\right)$ & 5.123 & 4.879 & & & & & \\
$\left(4^{4} D_{5 / 2}\right)$ & 5.161 & 4.885 & & & & & \\
$\left(4^{4} D_{7 / 2}\right)$ & 5.092 & 4.866 & & & & & \\
$\left(1^{4} F_{3 / 2}\right)$ & 4.467 & 4.348 & & & & & \\
$\left(1^{2} F_{5 / 2}\right)$ & 4.391 & 4.321 & & & & & \\
$\left(1^{4} F_{5 / 2}\right)$ & 4.429 & 4.328 & & & & \\
$\left(1^{4} F_{7 / 2}\right)$ & 4.360 & 4.303 & & & & \\
$\left(1^{2} F_{7 / 2}\right)$ & 4.353 & 4.296 & & & & \\
$\left(1^{4} F_{9 / 2}\right)$ & 4.292 & 4.274 & & & & \\
$\left(2^{4} F_{3 / 2}\right)$ & 4.802 & 4.593 & & & & \\
$\left(2^{2} F_{5 / 2}\right)$ & 4.726 & 4.569 & & & & \\
$\left(2^{4} F_{5 / 2}\right)$ & 4.764 & 4.575 & & & & \\
$\left(2^{4} F_{7 / 2}\right)$ & 4.695 & 4.553 & & & & \\
$\left(2^{2} F_{7 / 2}\right)$ & 4.688 & 4.547 & & & & \\
$\left(2^{4} F_{9 / 2}\right)$ & 4.627 & 4.527 & & & &
\end{tabular}

Our obtained orbital excited masses for $\Omega_{c c}$ show differences with Ref. [38], $1 P$ state $J^{P}=\frac{1}{2}^{-}$shows $25 \mathrm{MeV}, J^{P}=\frac{3}{2}^{-}$shows $16 \mathrm{MeV}$ and $J^{P}=\frac{5}{2}^{-}$ shows $32 \mathrm{MeV}, 2 P$ state $J^{P}=\frac{1}{2}^{-}$shows $6 \mathrm{MeV}$ (with [53]), $J^{P}=\frac{3}{2}^{-}$shows $15 \mathrm{MeV}$ (with [54]) difference. Our results for $3 P$ states masses are lower than in Ref. [38] in the range of $\approx 70 \mathrm{MeV}$. For $4 P$ state $J^{P}=\frac{1}{2}^{-}$shows $35 \mathrm{MeV}$, state $J^{P}=\frac{3}{2}^{-}$shows $9 \mathrm{MeV}$ and $J^{P}=\frac{5}{2}^{-}$shows $19 \mathrm{MeV}$ difference with Ref. [38]. Our $5 P$ states masses are higher than those in Ref. [38] in the range of $\approx 190 \mathrm{MeV}$.

Our outcome for $1 D$ state $J^{P}=\frac{3}{2}^{+}$shows $7 \mathrm{MeV}, J^{P}=\frac{5}{2}^{+}$shows $24 \mathrm{MeV}, J^{P}=\frac{7}{2}^{+}$shows $36 \mathrm{MeV}, 2 D$ state $J^{P}=\frac{3}{2}^{+}$shows $4 \mathrm{MeV}, J^{P}=\frac{5}{2}^{+}$ shows $16 \mathrm{MeV}$ and $J^{P}=\frac{7}{2}^{+}$shows $36 \mathrm{MeV}$ (with [38]) difference. For the $3 D-4 D$ states, the difference is 97 and $226 \mathrm{MeV}$ for $J^{P}=\frac{7}{2}^{+}$with Ref. [38]. 
TABLE V

The masses of orbital excited states of $\Omega_{b b}$ (in $\mathrm{GeV}$ ).

\begin{tabular}{|c|c|c|c|c|c|c|c|}
\hline State & Our calc. & [38] & [53] & [54] & [47] & [62] & Other \\
\hline$\left(1^{2} P_{1 / 2}\right)$ & 10.968 & 10.646 & 10.607 & 10.616 & 10.532 & & $10.519[55]$ \\
\hline$\left(1^{2} P_{3 / 2}\right)$ & 10.957 & 10.641 & 10.608 & 10.619 & 10.566 & $10.593 \pm 58$ & $10.520[49,50]$ \\
\hline$\left(1^{4} P_{1 / 2}\right)$ & 10.976 & 10.648 & & & & & \\
\hline$\left(1^{4} P_{3 / 2}\right)$ & 10.963 & 10.643 & & & & & $10.513[52]$ \\
\hline$\left(1^{4} P_{5 / 2}\right)$ & 10.956 & 10.637 & 10.808 & 10.766 & 10.798 & $10.700 \pm 60$ & \\
\hline$\left(2^{2} P_{1 / 2}\right)$ & 11.031 & 10.897 & 10.796 & 10.763 & 10.738 & & $10.683[55]$ \\
\hline$\left(2^{2} P_{3 / 2}\right)$ & 11.029 & 10.893 & 10.797 & 10.765 & 10.775 & & \\
\hline$\left(2^{4} P_{1 / 2}\right)$ & 11.039 & 10.899 & & & 10.924 & & \\
\hline$\left(2^{4} P_{3 / 2}\right)$ & 11.035 & 10.898 & & & 10.961 & & \\
\hline$\left(2^{4} P_{5 / 2}\right)$ & 11.019 & 10.888 & 11.028 & & & & \\
\hline$\left(3^{2} P_{1 / 2}\right)$ & 11.124 & 11.123 & 10.803 & & 11.083 & & \\
\hline$\left(3^{2} P_{3 / 2}\right)$ & 11.111 & 11.120 & 10.805 & & & & \\
\hline$\left(3^{4} P_{1 / 2}\right)$ & 11.131 & 11.125 & & & & & \\
\hline$\left(3^{4} P_{3 / 2}\right)$ & 11.119 & 11.122 & & & & & \\
\hline$\left(3^{4} P_{5 / 2}\right)$ & 11.112 & 11.177 & 11.059 & & & & \\
\hline$\left(4^{2} P_{1 / 2}\right)$ & 11.225 & 11.332 & & & & & \\
\hline$\left(4^{2} P_{3 / 2}\right)$ & 11.223 & 11.339 & & & & & \\
\hline$\left(4^{4} P_{1 / 2}\right)$ & 11.232 & 11.334 & & & & & \\
\hline$\left(4^{4} P_{3 / 2}\right)$ & 11.230 & 11.331 & & & & & \\
\hline$\left(4^{4} P_{5 / 2}\right)$ & 11.213 & 11.322 & & & & & \\
\hline$\left(5^{2} P_{1 / 2}\right)$ & 11.356 & 11.528 & & & & & \\
\hline$\left(5^{2} P_{3 / 2}\right)$ & 11.343 & 11.525 & & & & & \\
\hline$\left(5^{4} P_{1 / 2}\right)$ & 11.363 & 11.530 & & & & & \\
\hline$\left(5^{4} P_{3 / 2}\right)$ & 11.351 & 11.527 & & & & & \\
\hline$\left(5^{4} P_{5 / 2}\right)$ & 11.344 & 11.523 & & & & & \\
\hline$\left(1^{4} D_{1 / 2}\right)$ & 11.038 & 10.804 & & & & & \\
\hline$\left(1^{2} D_{3 / 2}\right)$ & 11.032 & 10.797 & & & & & \\
\hline$\left(1^{4} D_{3 / 2}\right)$ & 11.033 & 10.800 & & & & & \\
\hline$\left(1^{2} D_{5 / 2}\right)$ & 11.017 & 10.792 & 10.729 & 10.720 & & $10.858 \pm 77$ & \\
\hline$\left(1^{4} D_{5 / 2}\right)$ & 11.019 & 10.794 & & & & & \\
\hline$\left(1^{4} D_{7 / 2}\right)$ & 11.007 & 10.786 & & & & $10.964 \pm 80$ & \\
\hline$\left(2^{4} D_{1 / 2}\right)$ & 11.126 & 11.036 & & & & & \\
\hline$\left(2^{2} D_{3 / 2}\right)$ & 11.116 & 11.030 & & & & & \\
\hline$\left(2^{4} D_{3 / 2}\right)$ & 11.119 & 11.032 & & & & & \\
\hline$\left(2^{2} D_{5 / 2}\right)$ & 11.104 & 11.025 & 10.744 & 10.734 & & & \\
\hline$\left(2^{4} D_{5 / 2}\right)$ & 11.106 & 11.027 & & & & & \\
\hline$\left(2^{4} D_{7 / 2}\right)$ & 11.094 & 11.021 & & & & & \\
\hline
\end{tabular}


TABLE V

Continued.

\begin{tabular}{cccccccc}
\hline \hline State & Our calc. & {$[38]$} & {$[53]$} & {$[54]$} & {$[47]$} & {$[62]$} & Other \\
\hline$\left(3^{4} D_{1 / 2}\right)$ & 11.232 & 11.249 & & & & & \\
$\left(3^{2} D_{3 / 2}\right)$ & 11.223 & 11.244 & & & & & \\
$\left(3^{4} D_{3 / 2}\right)$ & 11.225 & 11.246 & & & & & \\
$\left(3^{2} D_{5 / 2}\right)$ & 11.210 & 11.240 & 10.937 & & & \\
$\left(3^{4} D_{5 / 2}\right)$ & 11.213 & 11.241 & & & & \\
$\left(3^{4} D_{7 / 2}\right)$ & 11.201 & 11.236 & & & & \\
$\left(4^{4} D_{1 / 2}\right)$ & 11.358 & 11.448 & & & & \\
$\left(4^{2} D_{3 / 2}\right)$ & 11.348 & 11.444 & & & & \\
$\left(4^{4} D_{3 / 2}\right)$ & 11.351 & 11.445 & & & & \\
$\left(4^{2} D_{5 / 2}\right)$ & 11.336 & 11.440 & & & & \\
$\left(4^{4} D_{5 / 2}\right)$ & 11.339 & 11.441 & & & \\
$\left(4^{4} D_{7 / 2}\right)$ & 11.327 & 11.437 & & & \\
$\left(1^{4} F_{3 / 2}\right)$ & 11.118 & 10.943 & & & & \\
$\left(1^{2} F_{5 / 2}\right)$ & 11.105 & 10.936 & & & & \\
$\left(1^{4} F_{5 / 2}\right)$ & 11.107 & 10.938 & & & \\
$\left(1^{4} F_{7 / 2}\right)$ & 11.094 & 10.932 & & & \\
$\left(1^{2} F_{7 / 2}\right)$ & 11.082 & 10.930 & & & \\
$\left(1^{4} F_{9 / 2}\right)$ & 11.073 & 10.924 & & & \\
$\left(2^{4} F_{3 / 2}\right)$ & 11.226 & 11.162 & & & \\
$\left(2^{2} F_{5 / 2}\right)$ & 11.210 & 11.155 & & & \\
$\left(2^{4} F_{5 / 2}\right)$ & 11.214 & 11.157 & & & \\
$\left(2^{4} F_{7 / 2}\right)$ & 11.202 & 11.151 & & & \\
$\left(2^{2} F_{7 / 2}\right)$ & 11.189 & 11.149 & & & \\
$\left(2^{4} F_{9 / 2}\right)$ & 11.179 & 11.144 & & &
\end{tabular}

Our result for $1 F$ state $J^{P}=\frac{7}{2}^{-}$is $30 \mathrm{MeV}$ lower than in Ref. [61] and for the $1 F-2 F$ states $J^{P}=\frac{9}{2}^{-}$are 18 and $100 \mathrm{MeV}$ higher than in Ref. [38]. For the ground and excited states of doubly heavy baryons $\left(\Omega_{c c}\right)$, the minimum and maximum percentage of relative error values are $0.03 \%$ and $3.662 \%$ between our calculations and the masses reported by Shah et al. [38].

Our estimated orbital excited masses of $\Omega_{b b}, 1 P$ state have difference in the range of $\approx 300 \mathrm{MeV}$ with other predictions. Our $2 P$ state $J^{P}=\frac{1}{2}^{-}$ and $J^{P}=\frac{3}{2}^{-}$are 135 and $136 \mathrm{MeV}$ higher than in Ref. [28] and $J^{P}=\frac{5}{2}^{-}$is $16 \mathrm{MeV}$ lower than Ref. [53], respectively. For $3 P$ state $J^{P}=\frac{1}{2}^{-}$and $J^{P}=\frac{3}{2}^{-}$ show only 1 and $8 \mathrm{MeV}$ difference with Ref. [38]. The reported mass of Ref. [53] for $J^{P}=\frac{5}{2}^{-}$state is $52 \mathrm{MeV}$ lower than our prediction. Our $4 P-5 P$, 
The masses of orbital excited states of $\Omega_{b c}$ (in GeV).

\begin{tabular}{|c|c|c|c|c|c|}
\hline State & Our calc. & [38] & State & Our calc. & [38] \\
\hline$\left(1^{2} P_{1 / 2}\right)$ & 7.476 & 7.386 & $\left(2^{2} D_{1 / 2}\right)$ & 7.806 & 7.843 \\
\hline$\left(1^{2} P_{3 / 2}\right)$ & 7.470 & 7.373 & $\left(2^{2} D_{3 / 2}\right)$ & 7.768 & 7.829 \\
\hline$\left(1^{4} P_{1 / 2}\right)$ & 7.490 & 7.392 & $\left(2^{4} D_{3 / 2}\right)$ & 7.792 & 7.834 \\
\hline$\left(1^{4} P_{3 / 2}\right)$ & 7.486 & 7.379 & $\left(2^{2} D_{5 / 2}\right)$ & 7.744 & 7.816 \\
\hline$\left(1^{4} P_{5 / 2}\right)$ & 7.451 & 7.363 & $\left(2^{4} D_{5 / 2}\right)$ & 7.767 & 7.821 \\
\hline$\left(2^{2} P_{1 / 2}\right)$ & 7.619 & 7.674 & $\left(2^{4} D_{7 / 2}\right)$ & 7.724 & 7.805 \\
\hline$\left(2^{2} P_{3 / 2}\right)$ & 7.595 & 7.664 & $\left(3^{2} D_{1 / 2}\right)$ & 8.017 & 8.088 \\
\hline$\left(2^{4} P_{1 / 2}\right)$ & 7.633 & 7.679 & $\left(3^{2} D_{3 / 2}\right)$ & 7.978 & 8.075 \\
\hline$\left(2^{4} P_{3 / 2}\right)$ & 7.610 & 7.669 & $\left(3^{4} D_{3 / 2}\right)$ & 8.002 & 8.079 \\
\hline$\left(2^{4} P_{5 / 2}\right)$ & 7.594 & 7.655 & $\left(3^{2} D_{5 / 2}\right)$ & 7.954 & 8.063 \\
\hline$\left(3^{2} P_{1 / 2}\right)$ & 7.782 & 7.935 & $\left(3^{4} D_{5 / 2}\right)$ & 7.976 & 8.068 \\
\hline$\left(3^{2} P_{3 / 2}\right)$ & 7.777 & 7.925 & $\left(3^{4} D_{7 / 2}\right)$ & 7.935 & 8.054 \\
\hline$\left(3^{4} P_{1 / 2}\right)$ & 7.796 & 7.939 & $\left(4^{2} D_{1 / 2}\right)$ & 8.266 & 8.317 \\
\hline$\left(3^{4} P_{3 / 2}\right)$ & 7.793 & 7.930 & $\left(4^{2} D_{3 / 2}\right)$ & 8.228 & 8.305 \\
\hline$\left(3^{4} P_{5 / 2}\right)$ & 7.758 & 7.918 & $\left(4^{4} D_{3 / 2}\right)$ & 8.252 & 8.309 \\
\hline$\left(4^{2} P_{1 / 2}\right)$ & 8.002 & 8.175 & $\left(4^{2} D_{5 / 2}\right)$ & 8.204 & 8.294 \\
\hline$\left(4^{2} P_{3 / 2}\right)$ & 7.978 & 8.167 & $\left(4^{4} D_{5 / 2}\right)$ & 8.229 & 8.298 \\
\hline$\left(4^{4} P_{1 / 2}\right)$ & 8.017 & 8.179 & $\left(4^{4} D_{7 / 2}\right)$ & 8.185 & 8.285 \\
\hline$\left(4^{4} P_{3 / 2}\right)$ & 7.994 & 8.171 & $\left(1^{4} F_{3 / 2}\right)$ & 7.792 & 7.742 \\
\hline$\left(4^{4} P_{5 / 2}\right)$ & 7.979 & 8.160 & $\left(1^{2} F_{5 / 2}\right)$ & 7.744 & 7.723 \\
\hline$\left(5^{2} P_{1 / 2}\right)$ & 8.242 & 8.400 & $\left(1^{4} F_{5 / 2}\right)$ & 7.768 & 7.728 \\
\hline$\left(5^{2} P_{3 / 2}\right)$ & 8.237 & 8.393 & $\left(1^{4} F_{7 / 2}\right)$ & 7.723 & 7.711 \\
\hline$\left(5^{4} P_{1 / 2}\right)$ & 8.256 & 8.404 & $\left(1^{2} F_{7 / 2}\right)$ & 7.720 & 7.705 \\
\hline$\left(5^{4} P_{3 / 2}\right)$ & 8.252 & 8.396 & $\left(1^{4} F_{9 / 2}\right)$ & 7.681 & 7.690 \\
\hline$\left(5^{4} P_{5 / 2}\right)$ & 8.218 & 8.386 & $\left(2^{4} F_{3 / 2}\right)$ & 8.001 & 7.965 \\
\hline$\left(1^{2} D_{1 / 2}\right)$ & 7.634 & 7.577 & $\left(2^{2} F_{5 / 2}\right)$ & 7.956 & 7.949 \\
\hline$\left(1^{2} D_{3 / 2}\right)$ & 7.597 & 7.561 & $\left(2^{4} F_{5 / 2}\right)$ & 7.978 & 7.953 \\
\hline$\left(1^{4} D_{3 / 2}\right)$ & 7.618 & 7.566 & $\left(2^{4} F_{7 / 2}\right)$ & 7.935 & 7.938 \\
\hline$\left(1^{2} D_{5 / 2}\right)$ & 7.571 & 7.547 & $\left(2^{2} F_{7 / 2}\right)$ & 7.931 & 7.934 \\
\hline$\left(1^{4} D_{5 / 2}\right)$ & 7.595 & 7.552 & $\left(2^{4} F_{9 / 2}\right)$ & 7.892 & 7.921 \\
\hline$\left(1^{4} D_{7 / 2}\right)$ & 7.552 & 7.534 & & & \\
\hline
\end{tabular}

states respectively, have $\approx 100 \mathrm{MeV}$ and $\approx 170 \mathrm{MeV}$ difference with Ref. [38]. Our outcomes for $1 D$ state $J^{P}=\frac{5}{2}^{+}$and $J^{P}=\frac{7}{2}^{+}$show difference of 159 and $43 \mathrm{MeV}$ with Ref. [62]. Our obtained masses for $2 D$ states are different from Ref. [38] predictions in the range of $\approx 80 \mathrm{MeV}$.

Our calculated masses for $3 D$ and $4 D$ states have difference in the range of $\approx 20$ and $\approx 100 \mathrm{MeV}$ lower than in Ref. [38]. Moving to $1 F$ state $J^{P}=\frac{7}{2}^{-}$ and $J^{P}=\frac{9}{2}^{-}$, the values are 36 and $148 \mathrm{MeV}$ higher than in Ref. [62]. 
Comparing our findings with the masses reported by Shah et al. [38], the minimum and maximum percentage of relative error values are $0.001 \%$ and $10.87 \%$ for the ground and excited states of doubly heavy baryons $\Omega_{b b}$.

The orbital mass spectrum of the third doubly heavy baryon, $\Omega_{b c}$, is predicted by Shah et al. [38] for the first time, as they believed. We also have not considered the diquark mechanism in our model and calculated orbital mass spectrum for the $\Omega_{b c}$ baryon. In the case of the doubly heavy baryon, $\Omega_{b c}$, our outcomes for the orbital excited masses are compared and discussed with Shah et al. [38] predictions in the following paragraph.

Our results for $1 P$ states are different from their predictions in the range of $\approx 90 \mathrm{MeV}$. We can easily observe that our calculations for $2 P, 1 D-2 D$ and $1 F-2 F$ states match with Shah's predictions. Our calculated masses for $3 P-5 P$ states are approximately 150,170 and $150 \mathrm{MeV}$ lover than their findings, respectively. Our results for $3 D$ and $4 D$ states are higher than their calculations, respectively, in the range of $\approx 90$ and $70 \mathrm{MeV}$. Comparing our calculations with the masses reported by Shah et al. [38], the minimum and maximum percentage of relative error values are $0.027 \%$ and $7.329 \%$ for the ground and excited states of doubly heavy baryons $\Omega_{b c}$.

\section{Conclusion}

In this study, we have computed the mass spectra of ground and excited states for doubly heavy $\Omega$ baryons by using a hypercentral constituent quark model. For this purpose, we have analytically solved the radial Schrödinger equation for three identical interacting particles under the effective hypercentral potential by using the Ansatz approach. Our proposed potential is regarded as a combination of the Coulomb-like term plus a linear confining term and the harmonic oscillator potential. We also added the first order correction and the spin-dependent part to the potential. Our model has succeeded to assign the $J^{P}$ values to the exited states of doubly heavy baryons $\left(\Omega_{c c}, \Omega_{b b}, \Omega_{b c}\right)$. Comparison of the results with other predictions revealed that they are in agreement and our proposed model can be useful for investigating the doubly heavy baryons states masses. For example, for the ground, radial and orbital excited states masses of doubly heavy $\Omega$ baryons, the minimum and the maximum percentage of relative error values are $1 \%$ and $6 \%$ between our calculations and the masses reported by Shah et al. [38]. As the final point, it should be clearly stated that the approach, despite its valuable predictions and results, does have its limitations including using the quasi-exact solutions and the fit process.

It is a great pleasure for the authors to thank the referee for the helpful comments. 


\section{REFERENCES}

[1] Z. Shah, A.K. Rai, Eur. Phys. J. C 77, 129 (2017).

[2] K.A. Olive et al., Chin. Phys. C 38, 090001 (2014).

[3] S. Koshkarev, V. Anikeev, Phys. Lett. B 765, 171 (2017) [arXiv: 1605.03070 [hep-p]].

[4] C. Patrignani et al. [Particle Data Group], Chin. Phys. C 40, 100001 (2016).

[5] M. Mattson et al. [SELEX Collaboration], Phys. Rev. Lett. 89, 112001 (2002).

[6] A. Ocherashvili et al. [SELEX Collaboration], Phys. Lett. B 628, 18 (2005).

[7] R. Aaij et al. [LHCb Collaboration], Phys. Rev. Lett. 118, 182001 (2017).

[8] D. Ebert, R.N. Faustov, V.O. Galkin, Phys. Rev. D 84, 014025 (2011).

[9] C. Garcia-Recio et al., Phys. Rev. D 87, 034032 (2013).

[10] W.H. Liang, C.W. Xiao, E. Oset, Phys. Rev. D 89, 054023 (2014).

[11] B. Chen, K.W. Wei, A. Zhang, Eur. Phys. J. A 51, 82 (2015).

[12] T. Yoshida et al., Phys. Rev. D 92, 114029 (2015).

[13] Ke-Wei Wei et al., Phys. Rev. D 95, 116005 (2017) [arXiv:609.02512 [hep-ph]].

[14] Y. Yamaguchi et al., Phys. Rev. D 91, 034034 (2015).

[15] Q. Mao et al., Phys. Rev. D 92, 114007 (2015).

[16] N. Isgur, G. Karl, Phys. Rev. D 20, 1191 (1979).

[17] M.M. Giannini, Rep. Prog. Phys. 54, 453 (1991).

[18] N. Salehi, A.A. Rajabi, Z. Ghalenovi, Acta Phys. Pol. B 42, 1247 (2011).

[19] Z. Shah, K. Thakkar, A. Kumar Rai, P.C. Vinodkumar, Chin. Phys. C 40, 123102 (2016).

[20] N. Salehi, H. Hassanabadi, Rom. Rep. Phys. 67, 403 (2015).

[21] L.A. Copley, G. Karl, E. Obryk, Phys. Lett. B 29, 117 (1969).

[22] R. Koniuk, N. Isgur, Phys. Rev. D 21, 1868 (1980).

[23] E. Santopinto, F. Iachello, M.M. Giannini, Eur. Phys. J. A 1, 307 (1998).

[24] M. Ferraris et al., Phys. Lett. B 364, 231 (1995).

[25] M.M. Giannini et al., Eur. Phys. J. A 12, 447 (2001).

[26] M. Campostrini, K. Moriarty, C. Rebbi, Phys. Rev. D 36, 3450 (1987).

[27] G.S. Bali, Phys. Rep. 343, 1 (2001).

[28] L.I. Abou-Salem, Adv. High Energy Phys. 2014, 196484 (2014).

[29] Z. Shah, K. Thakkar, A. Kumar Rai, P.C. Vinodkumar, Eur. Phys. J. A 52, 313 (2016).

[30] H. Garcilazo, J. Vijande, A. Valcarce, J. Phys. G 34, 961 (2007). 
[31] E. Santopinto, M.M. Giannini, F. Iachello, in: Symmetries in Science VII, B. Gruber (Ed.), Plenum Press, New York 1995, p. 445; F. Iachello, in: Symmetries in Science VII, B. Gruber (Ed.), Plenum Press, New York 1995, p. 213.

[32] K. Thakkar et al., Nucl. Phys. A 965, 57 (2017).

[33] Y. Koma, M. Koma, H. Wittig, Phys. Rev. Lett. 97, 122003 (2006).

[34] A.K. Rai, D.P. Rathaud, Eur. Phys. J. C 75, 462 (2015).

[35] N. Devlani, V. Kher, A.K. Rai, Eur. Phys. J. A 50, 154 (2014).

[36] Z. Shah, A.K. Rai, Eur. Phys. J. A 53, 195 (2017).

[37] Z. Shah, K. Thakkar, A.K. Rai, P.C. Vinodkumar, Chin. Phys. C 40, 123102 (2016).

[38] Z. Shah, K. Thakkar, A.K. Rai, Eur. Phys. J. C 76, 530 (2016).

[39] W. Lucha, F. Schoberls, Int. J. Mod. Phys. C 10, 607 (1997).

[40] J. Ballot, M. Fabre de la Ripelle, Ann. Phys. (N.Y.) 127, 62 (1980).

[41] N. Salehi, A.A. Rajabi, Eur. Phys. J. Plus 128, 27 (2013).

[42] U. Loring, K. Kretzschmar, B.Ch. Metsch, H.R. Petry, Eur. Phys. J. A 10, 309 (2001).

[43] N. Salehi, Adv. High Energy Phys. 2016, 5054620 (2016).

[44] E. Santopinto, Phys. Rev. C 72, 022201 (2005).

[45] A.A. Rajabi, N. Salehi, Iran. J. Phys. Res. 8, 169 (2008).

[46] A.P. Martynenko, Phys. Lett. B 663, 317 (2008).

[47] D. Ebert, R.N. Faustov, V.O. Galkin, A.P. Martynenko, Phys. Rev. D 66, 014008 (2002).

[48] F. Giannuzzi, Phys. Rev. D 79, 094002 (2009).

[49] Z.G. Wang, Eur. Phys. J. A 47, 267 (2010).

[50] Z.G. Wang, Eur. Phys. J. C 68, 459 (2010).

[51] T.M. Aliev, K. Azizi, M. Savci, Nucl. Phys. A 895, 59 (2012).

[52] T.M. Aliev, K. Azizi, M. Savci, J. Phys. G 40, 065003 (2013).

[53] T. Yoshida et al., Phys. Rev. D 92, 114029 (2015).

[54] W. Roberts, M. Pervin, Int. J. Mod. Phys. A 23, 2817 (2008).

[55] A. Valcarce, H. Garcilazo, J. Vijande, Eur. Phys. J. A 37, 217 (2008).

[56] K.U. Can, G. Erkol, M. Oka, T.T. Takahashi, Phys. Rev. D 92, 114515 (2015).

[57] P.P. Rubio, S. Collins, G.S. Baliy, Phys. Rev. D 92, 034504 (2015).

[58] Z.S. Brown, W. Detmold, S. Meinel, K. Orginos, Phys. Rev. D 90, 094507 (2014).

[59] Y. Namekawa et al., Phys. Rev. D 87, 094512 (2013).

[60] C. Alexandrou et al., Phys. Rev. D 90, 074501 (2014).

[61] K.-W. Wei, B. Chen, Phys. Rev. D 92, 076008 (2015). 
[62] K.-W. Wei et al., Phys. Rev. D 95, 116005 (2017)

[arXiv:1609.02512 [hep-ph]].

[63] C. Albertus, E. Hernández, J. Nieves, J.M. Verde-Velasco, Eur. Phys. J. A 32, 183 (2007).

[64] Z. Ghalenovi, A.A. Rajabi, S.-X. Qin, D.H. Rischke, Mod. Phys. Lett. A 29, 1450106 (2014).

[65] A. Bernotas, V. Simonis, Lithuanian. J. Phys. 49, 19 (2009).

[66] R. Roncaglia, D.B. Lichtenberg, E. Predazzi, Phys. Rev. D 52, 1722 (1995). 